\title{
Photochemical Dehalogenation of Aryl Halides: Importance of Halogen Bonding
}

Ayda Elhage, Paolo Costa, Amrah Nassim, Anabel E. Lanterna* and Juan C. Scaiano*

Department of Chemistry and Biomolecular Science, Centre for Advanced Materials Research (CAMaR), University of Ottawa, 10 Marie Curie, Ottawa, ON K1N 6N5, Canada. 
ABSTRACT. Upon UVA irradiation, aryl halides can undergo dehalogenation in presence of bases and methanol as a hydrogen donor. This catalyst-free photochemical dehalogenation is furnished through a facile radical-chain reaction under mild conditions. The chain reaction follows UVA irradiation of the reaction mixture in a transition metal-free environment. Mechanistic studies support a chain mechanism in which initiation involves absorption by a methoxide-bromoarene complex facilitated by halogen bonding interactions. The methoxide-bromine interaction leads to a weakened $\mathrm{Br}-\mathrm{C}$ bond that is prone to facile cleavage during the initiation and propagation steps. 


\section{INTRODUCTION}

The transformation of haloarenes into the corresponding arenes has great relevance to organic synthesis, industrial processes as well as environmental remediation. ${ }^{1}$ Conventional methods for dehalogenation of aryl halides usually involve high $\mathrm{H}_{2}$ pressures, reduction by tin or silicon hydrides, ${ }^{2}$ or catalytic systems including transition metals like $\mathrm{Pd},{ }^{3-7} \mathrm{Rh},{ }^{8} \mathrm{Ru},{ }^{9-10} \mathrm{Mo}^{11}, \mathrm{Co}^{12}, \mathrm{Ni}^{5}$,

${ }^{13}$ More recent examples study the use of light to catalyze the reaction in the presence of organic ${ }^{14}$ or inorganic ${ }^{15}$ photocatalysts. Although some recent examples suggest concerted mechanisms through hydrogen bonding formation, ${ }^{16}$ the majority of the photosensitized mechanisms are based on the aryl radical formation. ${ }^{17-21}$ Many have suggested that the presence of a strong base, usually $\mathrm{KO} t \mathrm{Bu}$ in $\mathrm{DMSO}$, it is involved it the photoinduced $\mathrm{Ar}-\mathrm{X}$ bond dissociation. ${ }^{22}$ However, a 2017 landmark study by Rossi et al. ${ }^{19}$ showed that light-induced eT reactions -where none of the isolated reagents can absorb the excitation light- can be photoinitiated by the formation of a dimsyl anion (deprotonation of DMSO in a highly basic medium, such as in the presence of $\mathrm{KO} t \mathrm{Bu}$ ). They demonstrated that this anion absorbs light into the visible region, and can initiate the eT process with aryl and alkyl halides via photoejection mechanism for the initial Proton-Electron-Transfer (PET) path. As they state, "dimsyl anion or its derivatives, rather than $-O t B u$ or $-H$ anion, may be responsible for starting the arylation reactions by photostimulated base-promoted homolytic aromatic substitution reaction (BHAS)." Here, we show that aryl halides can also be photochemically dehalogenated (under mild UV excitation) in the presence of a strong base, i.e. methoxide, without solvent assistance (Scheme 1). The key discovery in this contribution results from the initiation step - where the halogen bond formation activates the reagents towards long wavelength light absorption—, and the propagation step — where halogen bonding weakens the $\mathrm{C}-\mathrm{X}$ bond promoting dissociation. The product is furnished through hydrogen abstraction during 
an electron-transfer-catalyzed chain process (proton-coupled-electron transfer -PCET). ${ }^{23}$ This contrasts with the reaction under thermal conditions in the dark, where the same substrate undergoes a nucleophilic aromatic substitution $\left(\mathrm{S}_{\mathrm{N}} \mathrm{Ar}\right)$. The reaction is most favored with very strong bases and electron withdrawing group (EWG) substituents, such as in Scheme 2, that exemplifies the type of system examined here, illustrating the contrasting pathways observed thermally and photochemically. These findings constitute a new path to understand the photoinduced dehalogenation process, where the base-halogen interaction can play a crucial role.

Solvent-mediated photoinitiation

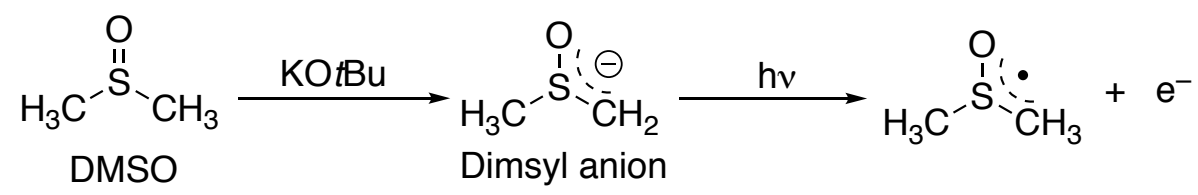

Base-mediated photoinitiation - this work

$$
\mathrm{Ar}-\mathrm{X} \stackrel{\text { base }}{\longrightarrow} \mathrm{Ar}-\mathrm{X}-\text { - -base } \stackrel{\mathrm{h} v}{\longrightarrow} \longrightarrow \text { Ar } \text { (other radicals) }
$$

Scheme 1. Photoinduced Ar-X bond dissociation: solvent-mediated photoinitiation ${ }^{19}$ and basemediated photoinitiation.
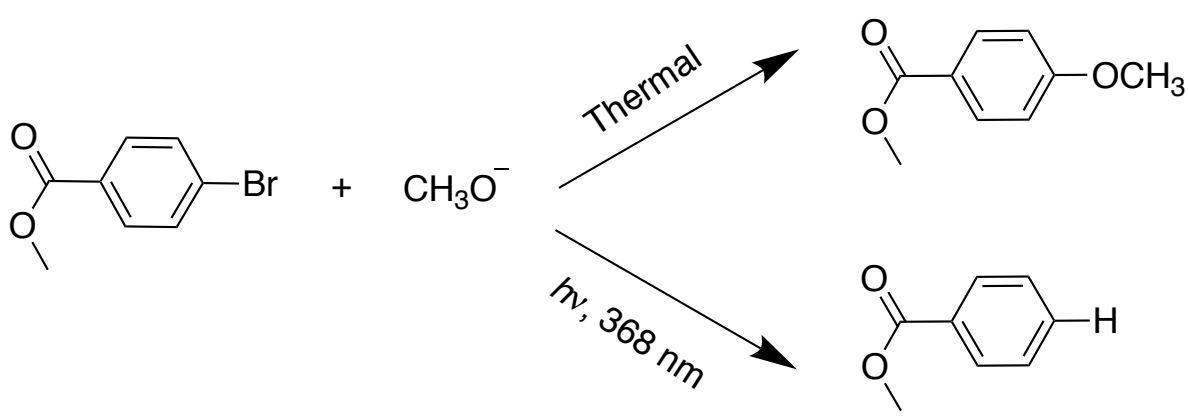

Scheme 2. Contrasting thermal and photochemical products in the reaction of 4-bromo methylbenzoate with methoxide in methanol.

METHODS 


\section{Experimental section}

All reagents and materials were purchased from Sigma Aldrich, unless otherwise stated, and used as received. Irgacure-2959 (I-2959) was a generous gift from BASF. Formaldehyde test is the MQuant ${ }^{\circledR}$ colorimetric test from Sigma.

UV-Vis spectroscopy was carried out in a Agilent Cary 60 using quartz cuvettes. Photoproduct yields were measured with a Perkin Elmer Clarus Gas Chromatographer (DB-5 column, $30 \mathrm{~m}$ length, $0.320 \mathrm{~mm}$ diameter, $0.25 \mu \mathrm{m}$ film) coupled to a Flame Ionization Detector (GC-FID) with helium carrier gas and dodecane as an external standard or an Agilent Gas Chromatographer coupled with mass spectrometry (GC-MS) and $t$-butylbenzene as an external standard. Lightemitting diodes from LedEngin were used for photochemical reactions in the UV region (centered at $368 \mathrm{~nm}$ ) and in the visible region (centered at $465 \mathrm{~nm}$ ).

\section{Photochemical dehalogenation}

In a dry and clean quartz tube $0.2 \mathrm{mmol}$ of 4-methyl-bromobenzoate and $0.6 \mathrm{mmol}$ of the base were suspended in $5 \mathrm{~mL}$ of HPLC methanol. The reaction mixture was sonicated for 15 min then purged with Argon for 10 min prior to irradiation with $368 \mathrm{~nm}$ LED working at 0.33 W.cm ${ }^{-2}$ under continuous stirring. Quantification of the product was done by GC-FID using dodecane as external standard.

\section{Formaldehyde test}

The formation of formaldehyde was detected by using the MQuant ${ }^{\circledR}$ test. Briefly, $0.5 \mathrm{~mL}$ of reaction mixture after $\mathrm{UV}$ irradiation were mixed together with $4 \mathrm{~mL}$ of milliQ water and 10 drops of the dye. Then, the strip was immersed in the solution and after $60 \mathrm{~s}$ the color change was evaluated. The test was repeated at least 3 times reaching saturation in all cases. Control test were performed using the reaction mixture before UV exposure. 


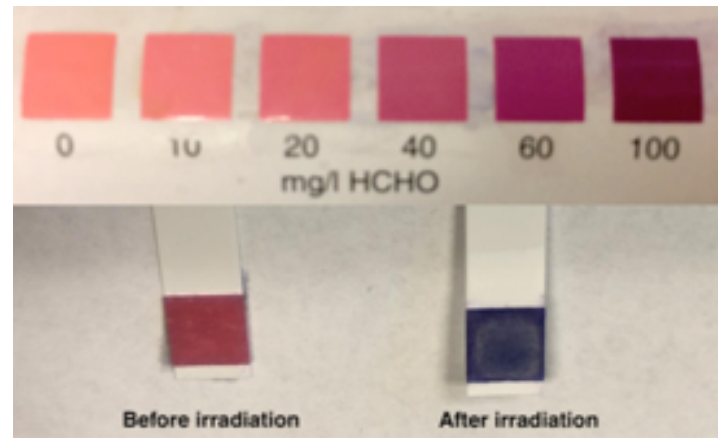

Figure 1. Formaldehyde test.

\section{Quantum yield}

Detailed calculation of the quantum yield can be found in the SI. The experimental procedure was as follows: ferrioxalate $6 \mathrm{mM}$ was prepared dissolving $60 \mathrm{mg}$ of potassium ferrioxalate in 20 $\mathrm{mL}$ of $0.05 \mathrm{M} \mathrm{H}_{2} \mathrm{SO}_{4}$ and phenanthroline $0.1 \%$ buffered solution was prepared by mixing $20 \mathrm{mg}$ of phenanthroline, $4.5 \mathrm{~g}$ of $\mathrm{CH}_{3} \mathrm{COONa} \cdot 3 \mathrm{H}_{2} \mathrm{O}$ in $20 \mathrm{~mL}$ of $0.5 \mathrm{M} \mathrm{H}_{2} \mathrm{SO}_{4}$. Both solutions are kept in brown bottles. For determination of the quantum yield, $4 \mathrm{~mL}$ of ferrioxalate solution were irradiated for $15 \mathrm{~min}$ in a photoreactor equipped with 14 UVA lamps prior to complexation with phenanthroline for determination of moles of ferrous ions produced in the solution. The photochemical dehalogenation was determined by mixing $0.2 \mathrm{mmol}$ of 4-methyl-bromobenzoate, 3 equiv. of $\mathrm{K}_{2} \mathrm{CO}_{3}$ in $4 \mathrm{~mL}$ of HPLC methanol in a clean dry quartz tube, then purged with argon prior to irradiation for $1 \mathrm{~h}$ in a photoreactor equipped with 14 UVA lamps.

\section{Computational details}

The DFT calculations presented in this work were performed using B3LYP and M06-2X density functionals $^{24-27}$ using def2-TZVP basis set. $^{28}$ Further, continuum solvation model was implemented, methanol was used as solvent via keywords SCRF=(IEFPCM, solvent=methanol). For B3LYP functional, D3 dispersion corrections have been included. ${ }^{29}$ All DFT computations 
were carried out with the Gaussian 09 software package. ${ }^{30}$ All the computed energies presented in this work include corrections for the zero-point vibrational energy unless stated otherwise.

\section{RESULTS AND DISCUSSION}

For the system shown in Scheme 1, the thermal reaction is completed within a few hours under reflux in methanol, while under a modest light intensity $\left(<1 \mathrm{Wcm}^{-2}, \mathrm{~T}<40^{\circ} \mathrm{C}\right)$ the photochemical product is obtained quantitatively in less than three hours (Table 1). This result is particularly interesting given that the initial reagents (base or benzyl halides) do not absorb light in the emission region of the light source. Naturally, light absorption is a requirement in the system, and therefore we decided to investigate the reaction in more detail, starting by the analysis of the absorption profile of the reaction mixture, i.e., methyl 4-bromobenzoate, various bases and methanol as solvent. As can be seen in Figure 2, the reaction mixture between methyl 4-bromobenzoate and a base shows a red-shifted absorption with a new small band around $350 \mathrm{~nm}$, which is responsible for the absorption of the LED light centered at $368 \mathrm{~nm}$. All bases show similar behavior (see SI), which is interpreted as all being simple methoxide sources. Moreover, the addition of base (MeONa) does not alter the absorption spectrum of methyl benzoate (Figure S2), which further proves that it is the interaction of the base and the halogen atom the responsible for the spectral change of the reaction mixture. Intrigued by the formation of this intermediate we use TimeDependent DFT (TD-DFT) calculations — using M06-2X level of theory ${ }^{31-34}$ - to simulate the absorption spectra of each methyl 4-halobenzoate before and after addition of $\mathrm{CH}_{3} \mathrm{O}^{-}$(Figure 2 and S3). It is commonly accepted that halogen atoms in organohalides present anisotropic electron density when they are covalently bonded to other atoms. Thus, the electrostatic potential around the covalent bond is highly negative whereas a positively charge region is formed over the halogen atom, known as the $\sigma$-hole..$^{5,35-36}$ This particular structural feature has attracted much attention ${ }^{5,}$ 
37-38 because it can interact with lone pairs (i.e., nucleophiles) forming halogen bonding. These halogen bonds $(\mathrm{XB})$ can influence conformation, binding and reactivity in the solution phase, ${ }^{37}$ including playing an important role in catalysis. ${ }^{38}$ As shown in Figure 3, the complex between methyl-4-bromobenzoate and methoxide involves a $\sigma$-hole interaction releasing $4.4 \mathrm{kcal} / \mathrm{mol}$ at B3LYP-D3/Def2TZVP level of theory. Similar interaction is predicted for chloro and iodo benzoates with binding energy of 1.4 and $10.5 \mathrm{kcal} / \mathrm{mol}$, respectively. It is also important to notice the $\mathrm{C}-\mathrm{X}$ bond is slightly elongated after the interaction with the methoxide is in place (Figure 3 and S3). Along with the halogen bond complex, other three local minima structures have been computed between methyl-4-bromobenzoate and methoxide (Figure S6). Among them, only the structure $b$ has higher binding energy than the halogen-bonding complex at both levels of theory (M06-2X and B3LYP-D3). Such structure corresponds to the anionic tetrahedral intermediate of the base-promoted hydrolysis of carboxylic esters, however, this intermediate would not furnish the photochemical product found. Indeed, the theoretical absorption spectrum of the tetrahedral intermediate is blue-shifted compared to that of 4-methyl-bromobenzoate (Figure S7).

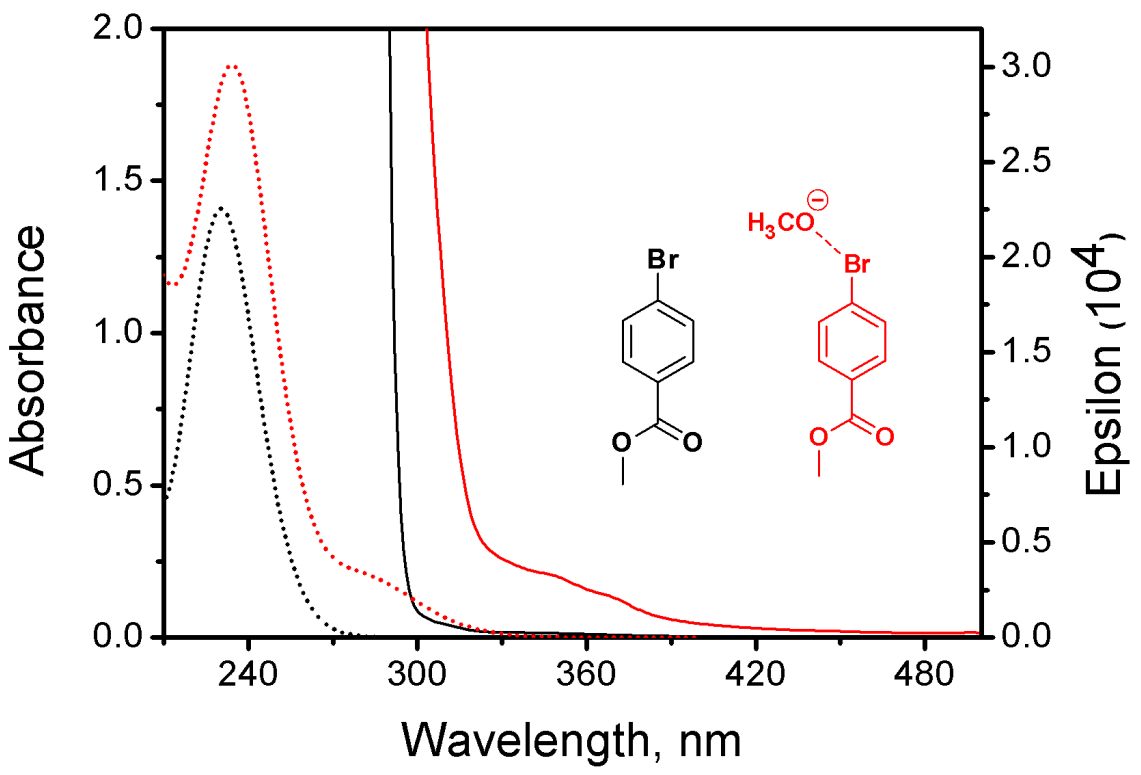


Figure 2. Absorption spectra for the reaction mixture in the absence of base (black solid line) and in the presence of 3 eq. of $\mathrm{NaOCH}_{3}$ (red solid line). Dashed lines represent the theoretical absorption spectra computed at the M06-2X/Def2TZVP, SCRF $=\left(\right.$ IEFPCM, solvent $\left.=\mathrm{CH}_{3} \mathrm{OH}\right)$ level of theory; calculated spectra also suggest a significant red shift.

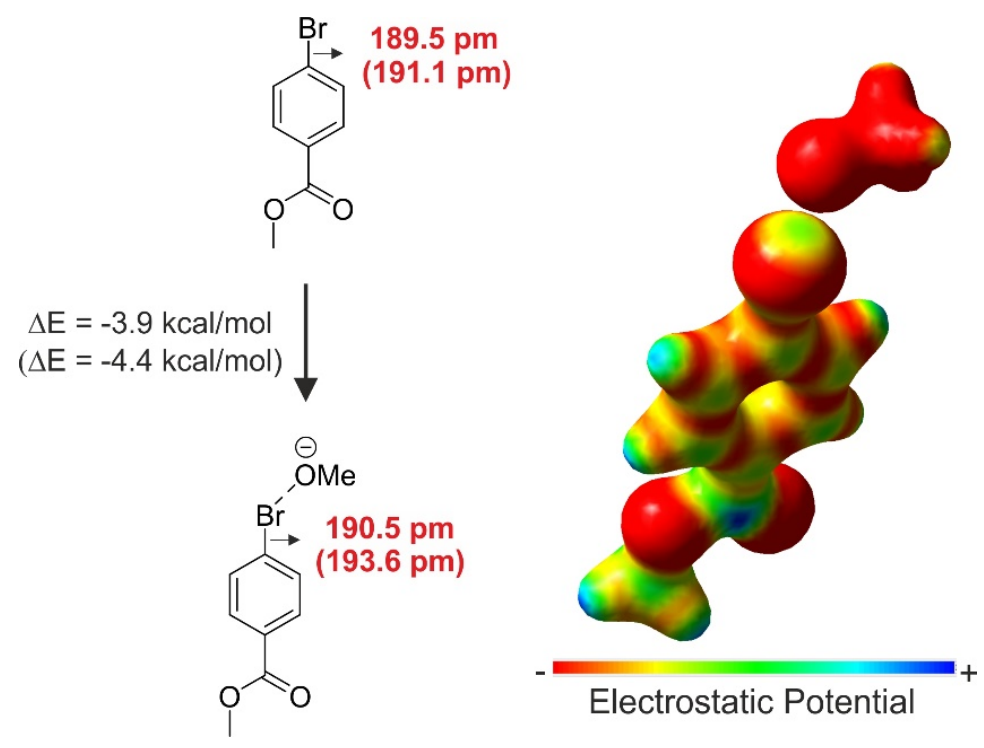

Figure 3. Optimized structure of methyl-4-bromobenzoate with and without methoxide at the M06-2X/Def2TZVP, SCRF $=\left(\right.$ IEFPCM, solvent $\left.=\mathrm{CH}_{3} \mathrm{OH}\right)$ level of theory. The $\mathrm{C}-\mathrm{Br}$ bond length is highlighted in red. Values in brackets were computed at the B3LYP-D3/Def2TZVP, $\mathrm{SCRF}=\left(\mathrm{IEFPCM}\right.$, solvent $\left.=\mathrm{CH}_{3} \mathrm{OH}\right)$ level of theory. $\Delta \mathrm{E}$ symbol represents the energy of the halogen bonding interaction. Plot of the electrostatic potential (isosurface value 0.02) is shown on the right (blue: positive potential, red: negative potential).

It is important to note that if no UV irradiation is applied to the system the reagent can be easily recovered i.e., by means of chromatography. Thus, the changes that occur upon mixing and lead to absorption at $350 \mathrm{~nm}$ must reflect a reversible equilibrium. 
In order to determine the nature of the photoinduced dehalogenation we performed different experiments summarized in table 1 (tables S1-S3). The photoinduced dehalogenation reaction works using UVA irradiation under inert atmosphere, atmospheric pressure, room temperature and in presence of bases - concentrations from 1 to 3 eq. show increased reaction yield (Table S2, entries ii-iv) — and methanol as a hydrogen donor. The reaction does not proceed in the absence of base, in the dark, or under visible light excitation. The solvent also plays an important role, the reaction works well in methanol, likely due to the in situ formation of methoxide. Ethanol and isopropanol also work, but yields are significantly lower (See SI). The reaction can work in toluene only in the presence of methanol and base, showing more soluble TMAOH can improve the reaction yields comparing to $\mathrm{K}_{2} \mathrm{CO}_{3}$ (Table S1). As shown in Table 1, the reaction is sensitive to the presence of radical quenchers such as oxygen, TEMPO or nitrobenzene (entries 9-11), and is accelerated in the presence of bromine (entry 3), a known radical initiator. ${ }^{39}$ However, the reaction does not proceed in the absence of base (entries 12-13), even using other radical initiators such as $\mathrm{Br}_{2}$ or Irgacure-2959 (Scheme S1) as shown in entries 12-13. It appears as if the only way to break the $\mathrm{C}-\mathrm{Br}$ bond is after it is debilitated by, for instance, the formation of halogen bonds, as shown in Figure 3. Finally, the quantum yield of the reaction was calculated as 43 , using ferrioxalate as actinometer. ${ }^{40-41}$ Based on all these evidence we can conclude that the dehalogenation reaction proceeds as a radical chain reaction.

Table 1. Reaction yields under standard conditions and with changes designed to test the reaction mechanism.

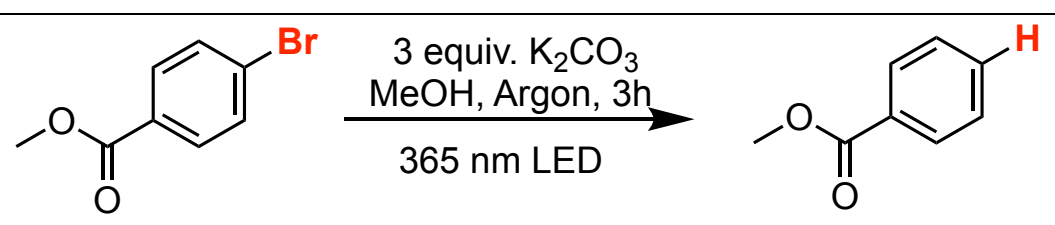




\begin{tabular}{lll}
\hline Entry & Changes to standard conditions & Yield \% ${ }^{\mathrm{a}}$ \\
\hline $\mathbf{1}$ & none & $\mathbf{9 8}$ \\
2 & $1 \mathrm{~h}$ irradiation & 52 \\
3 & $1 \mathrm{~h}$ irradiation, traces of $\mathrm{Br}_{2}$ & 79 \\
4 & $1 \mathrm{~h}$ irradiation, $0.71 \mathrm{~W} \mathrm{~cm}^{-2}$ & 70 \\
5 & $\mathrm{NaOCH}_{3}$ and no $\mathrm{K}_{2} \mathrm{CO}_{3}$ & 98 \\
6 & No base & traces \\
7 & Dark & 0 \\
8 & 465 nm LED irradiation & 0 \\
9 & Air & 0 \\
10 & 3 eq. of TEMPO & 0 \\
11 & 1 eq. of nitrobenzene & 0 \\
12 & No base, traces of Br & 0 \\
13 & No base, 2 eq. of photoinitiator & 0 \\
\hline
\end{tabular}

For the use of other bases see Tables S1-S2. See Figure 4 for the reaction dependence on light intensity. ${ }^{a}$ Conversions and yields determined by GC-FID using dodecane as an external standard. bI-2959.

The chain reaction proposed in order to rationalize the quantum yield of 43 is illustrated in Scheme 3, where the initiation step is favored by the XB formation between the base and the aryl halide. Further calculations suggest the $\mathrm{XB}$ weakens the $\mathrm{C}-\mathrm{X}$ bond favoring the dissociation in $50.1,40.2$ and $20.9 \mathrm{kcal} \mathrm{mol}^{-1}$ for $\mathrm{Cl}, \mathrm{Br}$ and I, respectively (Table S4). Note that the propagation is expected to yield formaldehyde in stoichiometric amount relative to ArH. Indeed, using a commercially available formaldehyde test, we could detect amounts higher than $100 \mathrm{mg} / \mathrm{L}$ of formaldehyde formed after reaction is completed (see Figure 1). As mentioned above, the reaction does not proceed in the absence of base, which could also support the role of the base as proton scavenger in the propagation step. Notice that the products of the initiation reaction are very minor 
and overlap with normal propagation products. Additionally, the chain length is expected to be much larger than 43 , as the quantum yield of photoinitiation is not expected to equal one. ${ }^{42}$ Further confirmation of the chain process is obtained from the negative curvature of the yield vs. irradiance plot (Figure 4), as in the case of chain reactions the anticipated second order termination causes reaction rates to depend upon $\mathrm{I}^{1 / 2}$.

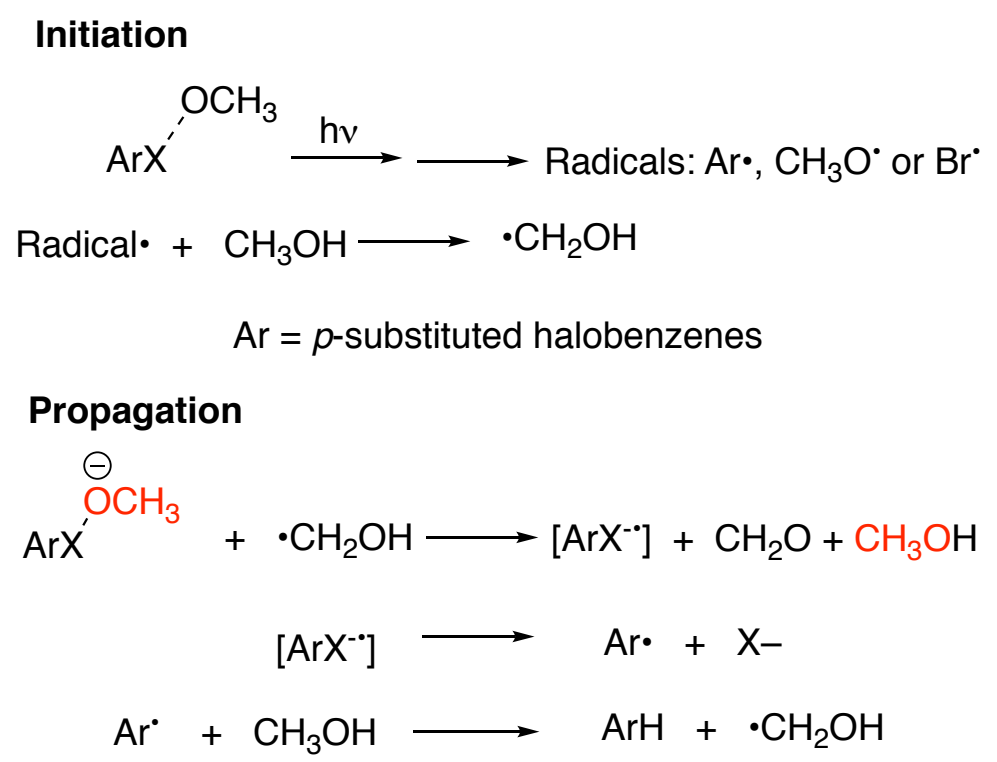

Scheme 3. Radical chain reaction proposed for the dehalogenation of $p$-substituted halobenzenes. 


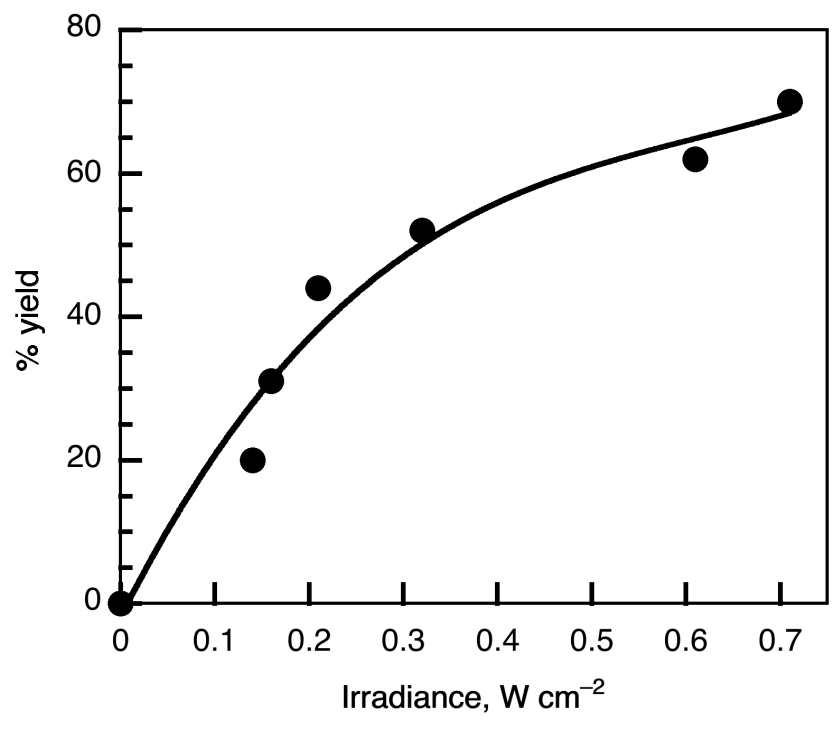

Figure 4. Dependence of the reaction yield with light intensity (368 nm) after irradiation for 45 minutes under otherwise standard conditions (see Table 1).

To further understand this base-halogen interaction we also evaluated replacing the fourth position substituent $\left(-\mathrm{C}(\mathrm{O}) \mathrm{OCH}_{3}, \sigma=+0.45^{43}\right)$ for a more EWG $\left(-\mathrm{CN}, \sigma=+0.66^{43}\right)$ or electron donor group, EDG, $\left(-\mathrm{OCH}_{3}, \sigma=-0.268^{43}\right)$. As shown in 
Table 2, the presence of an electron deficient group favors the reaction. Figure S4 clearly shows that the base-halogen interaction produces a greater red-shift of the aryl bromide absorption spectrum in the case of EWG; thus, it is not surprising that the reaction is more favored in those cases. We have also calculated the NBO charge distribution of these derivatives and found that the halogen bonding interaction is less favored for EDG (Figure S5), in agreement with our empirical findings. 
Table 2. Screening of the reaction with different 4-substituted bromobenzenes.

\begin{tabular}{lllll}
\hline Entry & $\mathrm{R}$ & Hammett Substituent Constants & \% Conversion & \% Yield $^{\mathrm{a}}$ \\
\hline $\mathrm{i}$ & $-\mathrm{CN}$ & +0.660 & 97 & 89 \\
ii & $-\mathrm{C}(\mathrm{O}) \mathrm{OCH}_{3}$ & +0.450 & $>99$ & $>98$ \\
iii & $-\mathrm{OCH}_{3}$ & -0.268 & 14 & $13^{\mathrm{b}}$ \\
\hline
\end{tabular}

Reaction conditions: $0.2 \mathrm{mmol}$ 4-substituted bromobenzene, 3 eq. MeONa, $5 \mathrm{~mL}$ of MeOH, 368 nm LED, Ar atmosphere. ${ }^{a}$ Conversion and yield determined by GC-MS using $t$-butylbenzene as an external standard. ${ }^{b} 24 \mathrm{~h}$ reaction, nothing detected after $6 \mathrm{~h}$ of irradiation.

\section{CONCLUSION}

While numerous strategies are known for reductive hydrodehalogenation, ${ }^{2}$ we believe the one reported here is exceptionally mild, facile and based on reagents that are transition metal-free and have excellent atom efficiency. It is also an illustration of how bond weakening induced by $\boldsymbol{\sigma}$ holes and halogen bonding can play a major role in organic methods.

In summary, we have described how the photochemical behavior of aryl halides in the presence of a nucleophile can furnish the dehalogenation product quantitatively through a radical chain reaction. The mechanism is surprisingly different from the $\mathrm{S}_{\mathrm{N}} \mathrm{Ar}$ taking place under dark conditions. Experimental and computational analysis of the absorption profile of the reaction mixture, together with mechanistic studies, suggest the reversible formation of a complex species, more likely stabilized by XB formation, that weakens the $\mathrm{C}-\mathrm{X}$ bond facilitating the radical reaction initiation. Additionally, the base acts as a proton scavenger that can also assist in the propagation step, as the reaction can be regarded as a PCET process.

ASSOCIATED CONTENT 
Supporting Information. More experimental and computational details are shown in the SI. The Supporting Information is available free of charge on the ACS Publications website.

\section{AUTHOR INFORMATION}

\section{Corresponding Authors}

*JCS: jscaiano@uottawa.ca*AEL: alantern@uottawa.ca

\section{Notes}

The authors declare no competing financial interests.

\section{ACKNOWLEDGMENT}

This work was supported by the Natural Sciences and Engineering Research Council of Canada, the Canada Foundation for Innovation, the Canada Re-search Chairs Program, Canada's International Development Research Centre (IDRC) and the German National Academy of Sciences Leopoldina (Grant No. LPDS 2017-15 to P.C.). The authors are grateful to Dr. Victoria Benavente Llorente for her generous assistance performing electrochemical measurements.

\section{REFERENCES}

1. Sadowsky, D.; McNeill, K.; Cramer, C. J., Dehalogenation of Aromatics by Nucleophilic Aromatic Substitution. Environ. Sci.Technol. 2014, 48, 10904-10911.

2. $\quad$ Alonso, F.; Beletskaya, I. P.; Yus, M., Metal-Mediated Reductive Hydrodehalogenation of Organic Halides. Chem. Rev. 2002, 102, 4009-4091.

3. Guo, X.; Yu, C.; Yin, Z.; Sun, S.; Seto, C. T., Hydrodehalogenation of Polyhalogenated Aromatics Catalyzed by NiPd Nanoparticles Supported on Nitrogen-Doped Graphene. ChemSusChem 2018, 11, 1617-1620.

4. $\quad$ Chen, Y. L.; Xiong, L.; Song, X. N.; Wang, W. K.; Huang, Y. X.; Yu, H. Q., Electrocatalytic Hydrodehalogenation of Atrazine in Aqueous Solution by $\mathrm{Cu} @ \mathrm{Pd} / \mathrm{Ti}$ Catalyst. Chemosphere 2015, 125, 57-63.

5. Cavallo, G.; Metrangolo, P.; Milani, R.; Pilati, T.; Priimagi, A.; Resnati, G.; Terraneo, G., The Halogen Bond. Chem. Rev. 2016, 116, 2478-2601. 
6. Zahran, E. M.; Bedford, N. M.; Nguyen, M. A.; Chang, Y. J.; Guiton, B. S.; Naik, R. R.; Bachas, L. G.; Knecht, M. R., Light-Activated Tandem Catalysis Driven by Multicomponent Nanomaterials. J. Am. Chem. Soc. 2014, 136, 32-5.

7. Bhattacharjya, A.; Klumphu, P.; Lipshutz, B. H., Ligand-Free, Palladium-Catalyzed Dihydrogen Generation from TMDS: Dehalogenation of Aryl Halides on Water. Org. Lett. 2015, $17,1122-1125$.

8. $\quad$ Katayama, Y.; Aoyagi, M.; Matsumoto, T.; Harada, H.; Simion, A. M.; Egashira, N.; Mitoma, Y.; Simion, C., Hydrodehalogenation of Hexachloro- and Hexabromobenzene by Metallic Calcium in Ethanol, in the Presence of Rh/C Catalyst. Environ. Sci. Pollut. Res. Int. 2017, 24, 591-597.

9. You, T. J.; Wang, Z. R.; Chen, J. J.; Xia, Y. Z., Transfer Hydro-Dehalogenation of Organic Halides Catalyzed by Ruthenium(II) Complex. J. Org. Chem. 2017, 82, 1340-1346.

10. Narayanam, J. M.; Tucker, J. W.; Stephenson, C. R., Electron-Transfer Photoredox Catalysis: Development of a Tin-Free Reductive Dehalogenation Reaction. J. Am. Chem. Soc. 2009, 131, 8756-7.

11. Tsurugi, H.; Hayakawa, A.; Kando, S.; Sugino, Y.; Mashima, K., Mixed-Ligand Complexes of Paddlewheel Dinuclear Molybdenum as Hydrodehalogenation Catalysts for Polyhaloalkanes. Chem. Sci. 2015, 6, 3434-3439.

12. Sahoo, B.; Surkus, A. E.; Pohl, M. M.; Radnik, J.; Schneider, M.; Bachmann, S.; Scalone, M.; Junge, K.; Beller, M., A Biomass-Derived Non-Noble Cobalt Catalyst for Selective Hydrodehalogenation of Alkyl and (Hetero)Aryl Halides. Angew. Chem. Int. Ed. Engl. 2017, 56, 11242-11247.

13. Rettenmeier, C. A.; Wenz, J.; Wadepohl, H.; Gade, L. H., Activation of Aryl Halides by Nickel(I) Pincer Complexes: Reaction Pathways of Stoichiometric and Catalytic Dehalogenations. Inorg. Chem. 2016, 55, 8214-24.

14. Gong, H. X.; Cao, Z.; Li, M. H.; Liao, S. H.; Lin, M. J., Photoexcited Perylene Diimide Radical Anions for the Reduction of Aryl Halides: A Bay-Substituent Effect. Org. Chem. Front. 2018, 5, 2296-2302.

15. Wang, Y. Y.; Zhu, Q.; Wei, Y.; Gong, Y. J.; Chen, C. C.; Song, W. J.; Zhao, J. C., Catalytic Hydrodehalogenation over Supported Gold: Electron Transfer Versus Hydride Transfer. Appl. Catal. B 2018, 231, 262-268.

16. Cao, D.; Yan, C.; Zhou, P.; Zeng, H.; Li, C.-J., Hydrogen Bonding Promoted Simple and Clean Photo-Induced Reduction of C-X Bond with Isopropanol. Chem. Commun. 2019, 55, 767770.

17. Arceo, E.; Montroni, E.; Melchiorre, P., Photo-Organocatalysis of Atom-Transfer Radical Additions to Alkenes. Angew. Chem. Int. Edit. 2014, 53, 12064-12068.

18. Poelma, S. O.; Burnett, G. L.; Discekici, E. H.; Mattson, K. M.; Treat, N. J.; Luo, Y.; Hudson, Z. M.; Shankel, S. L.; Clark, P. G.; Kramer, J. W., et al., Chemoselective Radical Dehalogenation and $\mathrm{C}-\mathrm{C}$ Bond Formation on Aryl Halide Substrates Using Organic Photoredox Catalysts. J. Org. Chem. 2016, 81, 7155-7160.

19. Buden, M. E.; Bardagi, J. I.; Puiatti, M.; Rossi, R. A., Initiation in Photoredox C-H Functionalization Reactions. Is Dimsyl Anion a Key Ingredient? J. Org. Chem. 2017, 82, 83258333.

20. Discekici, E. H.; Treat, N. J.; Poelma, S. O.; Mattson, K. M.; Hudson, Z. M.; Luo, Y.; Hawker, C. J.; de Alaniz, J. R., A Highly Reducing Metal-Free Photoredox Catalyst: Design and Application in Radical Dehalogenations. Chem. Commun. 2015, 51, 11705-11708. 
21. Bardagi, J. I.; Ghosh, I.; Schmalzbauer, M.; Ghosh, T.; König, B., Anthraquinones as Photoredox Catalysts for the Reductive Activation of Aryl Halides. Eur. J. Org. Chem. 2018, $2018,34-40$.

22. Qiu, G. Y. S.; Li, Y. W.; Wu, J., Recent Developments for the Photoinduced Ar-X Bond Dissociation Reaction. Org. Chem. Front. 2016, 3, 1011-1027.

23. Rossi, R. A.; Pierini, A. B.; Penenory, A. B., Nucleophilic Substitution Reactions by Electron Transfer. Chem. Rev. 2003, 103, 71-167.

24. Vosko, S. H.; Wilk, L.; Nusair, M., Accurate Spin-Dependent Electron Liquid Correlation Energies for Local Spin Density Calculations: A Critical Analysis. Can. J. Phys. 1980, 58, 1200-1211.

25. Lee, C. T.; Yang, W. T.; Parr, R. G., Development of the Colle-Salvetti CorrelationEnergy Formula into a Functional of the Electron-Density. Phys. Rev. B 1988, 37, 785-789. 26. Miehlich, B.; Stoll, H.; Savin, A., A Correlation-Energy Density Functional for Multideterminantal Wavefunctions. Mol. Phys. 1997, 91, 527-536.

27. Zhao, Y.; Truhlar, D. G., The M06 Suite of Density Functionals for Main Group Thermochemistry, Thermochemical Kinetics, Noncovalent Interactions, Excited States, and Transition Elements: Two New Functionals and Systematic Testing of Four M06-Class Functionals and 12 Other Functionals. Theor. Chem. Acc. 2008, 120, 215-241.

28. Weigend, F.; Haser, M.; Patzelt, H.; Ahlrichs, R., Ri-Mp2: Optimized Auxiliary Basis Sets and Demonstration of Efficiency. Chem. Phys. Lett. 1998, 294, 143-152.

29. Grimme, S.; Antony, J.; Ehrlich, S.; Krieg, H., A Consistent and Accurate Ab Initio Parametrization of Density Functional Dispersion Correction (DFT-D) for the 94 Elements H-Pu. Journal of Chemical Physics 2010, 132.

30. Gaussian 09, Revision E.01, M. J. Frisch, G. W. Trucks, H. B. Schlegel, G. E. Scuseria, M. A. Robb, J. R. Cheeseman, G. Scalmani, V. Barone, G. A. Petersson, H. Nakatsuji, X. Li, M. Caricato, A. Marenich, J. Bloino, B. G. Janesko, R. Gomperts, B. Mennucci, H. P. Hratchian, J. V. Ortiz, A. F. Izmaylov, J. L. Sonnenberg, D. Williams-Young, F. Ding, F. Lipparini, F. Egidi, J. Goings, B. Peng, A. Petrone, T. Henderson, D. Ranasinghe, V. G. Zakrzewski, J. Gao, N. Rega, G. Zheng, W. Liang, M. Hada, M. Ehara, K. Toyota, R. Fukuda, J. Hasegawa, M. Ishida, T. Nakajima, Y. Honda, O. Kitao, H. Nakai, T. Vreven, K. Throssell, J. A. Montgomery, Jr., J. E. Peralta, F. Ogliaro, M. Bearpark, J. J. Heyd, E. Brothers, K. N. Kudin, V. N. Staroverov, T. Keith, R. Kobayashi, J. Normand, K. Raghavachari, A. Rendell, J. C. Burant, S. S. Iyengar, J. Tomasi, M. Cossi, J. M. Millam, M. Klene, C. Adamo, R. Cammi, J. W. Ochterski, R. L. Martin, K. Morokuma, O. Farkas, J. B. Foresman, and D. J. Fox, Gaussian, Inc., Wallingford CT, 2016. 31. Costa, P.; Sander, W., Hydrogen Bonding Switches the Spin State of Diphenylcarbene from Triplet to Singlet. Angew. Chem. Int. Edit. 2014, 53, 5122-5125.

32. Henkel, S.; Costa, P.; Klute, L.; Sokkar, P.; Fernandez-Oliva, M.; Thiel, W.; SanchezGarcia, E.; Sander, W., Switching the Spin State of Diphenylcarbene Via Halogen Bonding. J. Am. Chem. Soc. 2016, 138, 1689-1697.

33. Henkel, S.; Trosien, I.; Mieres-Perez, J.; Lohmiller, T.; Savitsky, A.; Sanchez-Garcia, E.; Sander, W., Reactions of Cyclopentadienylidenes with Cf3i: Electron Bond Donation Versus Halogen Bond Donation of the Iodine Atom. J. Org. Chem. 2018, 83, 7586-7592.

34. Kozuch, S.; Martin, J. M. L., Halogen Bonds: Benchmarks and Theoretical Analysis. J. Chem. Theory Comput. 2013, 9, 1918-1931.

35. Clark, T., Halogen Bonds and Sigma-Holes. Faraday Discuss. 2017, 203, 9-27. 
36. Legon, A. C., The Halogen Bond: An Interim Perspective. Phys. Chem. Chem. Phys. 2010, $12,7736-7747$.

37. Beale, T. M.; Chudzinski, M. G.; Sarwar, M. G.; Taylor, M. S., Halogen Bonding in Solution: Thermodynamics and Applications. Chem. Soc. Rev. 2013, 42, 1667-1680.

38. Gilday, L. C.; Robinson, S. W.; Barendt, T. A.; Langton, M. J.; Mullaney, B. R.; Beer, P. D., Halogen Bonding in Supramolecular Chemistry. Chem. Rev. 2015, 115, 7118-7195.

39. Smith, M., March's Advanced Organic Chemistry : Reactions, Mechanisms and Structure; Hoboken John Wiley \& Sons, Inc.: Hoboken, 2007.

40. Montalti, M.; Credi, A.; Prodi, L.; Gandolfi, M. T., Handbook of Photochemistry. , 3rd ed.; Boca Raton: CRC/Taylor \& Francis, (C2006., 2006.

41. Hatchard, C. G.; Parker, C. A., A New Sensitive Chemical Actinometer. II. Potassium Ferrioxalate as a Standard Chemical Actinometer. Proc. R. Soc. London, Ser. A 1956, 235, 518536.

42. The quantum yield of the reaction is the product of the quantum yield of initiation times the chain length.

43. Leffler, J. E.; Grunwald, E., Rates and Equilibria of Organic Reactions as Treated by Statistical, Thermodynamic, and Extrathermodynamic Methods; New York, Wiley 1963: New York, 1963. 


\section{TOC GRAPHIC}

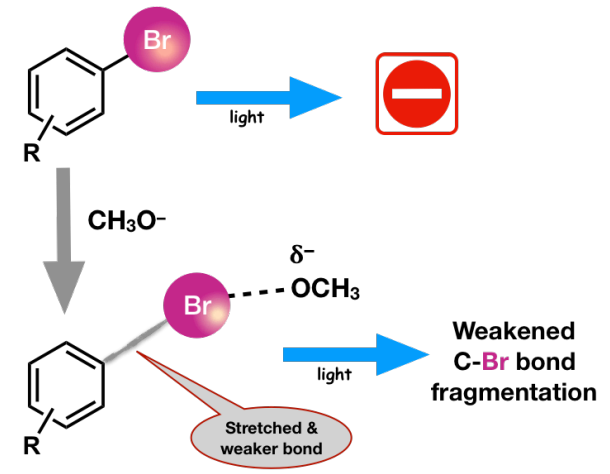

